\title{
Vibrotactile pattern isolation/integration
}

\author{
JAMES C. CRAIG \\ Indiana University, Bloomington, Indiana
}

\begin{abstract}
Temporal integration has been cited as a major factor in temporal masking. Two experiments were designed to examine the conditions under which temporal integration may aid or hinder the perception of vibrotactile spatial patterns. In Experiment 1, the subject's task was to discriminate between pairs of patterns. Each pattern was composed of two temporally separated pattern elements. When the task required the subjects to perceive the individual pattern elements, performance improved with temporal isolation-that is, performance improved as the temporal separation between the elements increased. In a second task, when the discrimination could be based on either the overall pattern shape or the pattern elements, temporal integration appeared to improve performancethat is, performance improved as the temporal separation decreased. In Experiment 2, an identification task was used. Several factors appeared to determine whether temporal integration aided or hindered pattern identification. When pattern elements similar to those in Experiment 1 were tested, performance improved with increasing temporal separation (isolation). A single function was fit to the discrimination (isolation) and identification (isolation) results. Whether temporal integration aids or hinders pattern perception appears to depend on pattern shape, the pattern elements, and the nature of the task.
\end{abstract}

More than 20 years ago, Kirman wrote an influential review article on the tactile communication of speech (Kirman, 1973). In that review, he discussed a number of limitations on the perception of complex patterns by the skin. One of the major limitations is temporal masking, in which a target pattern may be interfered with by the presentation of a second pattern in close temporal proximity to the first pattern. In dealing with the issue of temporal masking, Kirman considered two approaches to the communication of information to the skin. In the first approach, investigators may try to minimize masking by separating patterns, either spatially, temporally, or both. In the second approach, masking is viewed not so much as something to be avoided but rather as reflecting processes by which elements may interact and the relationships among these elements revealed. Kirman was specifically concerned with the communication of speech information to the skin and, thus, considered how phonemes (the elements) might combine to form higher linguistic units, such as words and sentences. According to Kirman, the focus in communicating information to the skin should shift from trying to devise stimulus conditions that isolate individual elements and preserve their distinctiveness to examining conditions in which these elements could combine and form larger, identifiable perceptual units.

Twenty years later, Mahar and Mackenzie (1993), in considering these ideas, framed the issue in terms of two

This research was supported by National Institutes of Health, Grant DC00095, National Institute on Deafness and Other Communication Disorders. The author thanks Roger Rhodes for his assistance in these experiments. Correspondence concerning this article should be addressed to J. C. Craig, Department of Psychology, Indiana University, Bloomington, IN 47405 (e-mail: craigj@indiana.edu). competing views-isolation and integration. According to the isolation hypothesis, accurate perception of tactile patterns results when the mutual interaction between successive pattern elements is reduced or, in Kirman's (1973) terms, when masking is minimized. The integration hypothesis takes the point of view that reducing temporal and spatial separation between elements may aid pattern perception by increasing temporal integration. The integrated pattern may be more perceptible, because particular relationships between pattern elements are revealed.

There is considerable evidence that, when two spatial patterns are presented in close spatial and temporal proximity, the result is an integrated pattern. For example, if subjects are presented a vertical line down the middle of their fingerpad, followed by a horizontal line, they may report feeling a cross rather than two lines. The onsets between two patterns must be as much as $70 \mathrm{msec}$ apart before subjects report perceiving two patterns and as much as $121 \mathrm{msec}$ apart before subjects report that the two patterns are perceived as discrete (Craig, 1996). Temporal integration, as the term will be used in the present context, is the process by which elements of patterns, presented in close temporal and spatial proximity to one another, form a single representation.

Temporal integration has been studied in several contexts, including temporal masking. If a target and nontarget pattern are presented in close temporal proximity, subjects may be unable to correctly identify the target because features of the target and the nontarget form a single pattern (Evans, 1987; Evans \& Craig, 1986). Under such circumstances, subjects will often respond that they felt a composite pattern, a pattern that consisted of the features of the target and of the nontarget added together. As the time between the two patterns increases, particu- 
larly to $100 \mathrm{msec}$, the amount of masking decreases, and composite responses become less likely as well. If subjects are asked to judge the number of line segments composing a target pattern, they show a marked tendency to overestimate the number of line segments when a second pattern is presented in close temporal proximity to the pattern being judged. Presumably subjects are integrating features from the target and the nontarget patterns, which, in turn, leads to the overestimation of the number of lines in the target pattern (Evans \& Craig, 1986). Again, as the temporal separation increases to $100 \mathrm{msec}$, the tendency to overestimate declines. These results indicate how difficult it can be for subjects to process separately two patterns that have been combined by temporal integration. In those situations in which subjects responded with composite patterns, they knew that, on every trial, two patterns were presented. Subjects also received trial-bytrial feedback. Yet, frequently they responded as though they had received only one pattern. Similarly, subjects in the estimation task also knew that two patterns were being presented yet were unable to avoid overestimating the number of lines in the target pattern. These results also indicate how integration may reduce the accurate perception of spatial patterns.

In view of the strong tendency for patterns to be integrated, the issue raised by Kirman (1973) and by Mahar and Mackenzie (1993) can be addressed, in part, by asking whether there are stimulus conditions and tasks in which integration improves performance and other stimulus conditions and tasks in which integration degrades performance - that is, in which isolation aids performance. As noted, when the task is one of identifying a target pattern in the presence of a second, nontarget pattern, the general result is that target identification is interfered with. It is likely that some of this interference effect results from temporal integration of the target and the nontarget, although other factors, such as response competition, may be responsible as well (Craig, 1996; Craig \& Evans, 1995).

It is more difficult to find tasks in which temporal integration is helpful to pattern perception. One study that did report improved performance for integrated patterns used letters of the alphabet. In this study, the subjects attempted to identify one of five letters of the alphabet (Craig, 1982). The letters were divided in half, and the subjects were presented the two halves of the letter sequentially. The temporal separations between halves ranged from 0 - to more than 200-msec stimulus onset asynchrony (SOA). Identification performance was best at 0 -msec SOA, the point at which temporal integration should be maximal, and declined from there to $50-100 \mathrm{msec}$. Had the task been to identify the individual letter halves - a typical masking paradigm and one that favors isolation-performance presumably would have been poor at $0 \mathrm{msec}$ and would have improved as the temporal separation increased.

One of the aims of the present study was to examine integration and isolation with similar tasks and stimuli. This aim is closely related to the study by Mahar and Mackenzie (1993). They developed tasks that they thought might reveal the beneficial effects of integration and the beneficial effects of isolation. The tasks involved tactile patterns that were delivered to the forearm by means of three tactile transducers. In one task, the subject was to identify one of the pattern elements - specifically, to select the vibrator at which the most intense stimulus was presented. This task was designed to favor isolation; it was predicted that greater temporal and spatial separation of the pattern elements would improve performance. The results supported this conclusion. The subjects' accuracy increased as temporal separation increased from 94 to $450 \mathrm{msec}$ and as spatial separation of the tactile transducers increased from 1.6 to $5.0 \mathrm{~cm}$.

The second task required subjects to discriminate between pairs of patterns. The patterns were generated in a manner similar to that in the first task. To perform this task, the subjects simply indicated whether the pair of patterns were the same or different, rather than identifying a particular pattern element. Because the subjects did not have to focus on each of the three vibratory signals composing the pattern and could instead attend to the pattern as a whole, this task was thought to favor the integration of stimuli. Hence, performance might be expected to improve as temporal and spatial separation was reduced. Contrary to the prediction, performance declined with decreasing temporal separation, as it had in the identification task. There was no significant effect of spatial separation, although the results were in the direction of decreasing spatial separation producing poorer rather than better discriminability.

A second aim of the present study was to see the extent to which one could view isolation as simply the mirror image of integration. Presumably, the extent to which two patterns can be isolated is simply the extent to which they are not integrated. Previous studies have not used similar tasks and similar stimuli under conditions in which these two processes might be evident. A third aim was to see the extent to which the results obtained in a discrimination task (Experiment 1) that involved temporal interactions could be used to predict the results in an identification task (Experiment 2).

\section{EXPERIMENT 1}

The rationale for Experiment 1 was to use a single task (discrimination) that could be considered neutral with regard to isolation and integration but to select conditions that favored either integration or isolation. As Mahar and Mackenzie (1993) pointed out, discrimination does not rely heavily on subjects' experience with particular patterns. Also, discrimination tasks require no label for the pattern, no verbal description of the pattern, and no assumptions about what the pattern will feel like to the subjects.

The first set of measurements in Experiment 1 followed the general framework suggested by Mahar and Mackenzie (1993): Set up a task in which increasing separation between pattern elements might lead to improved dis- 
criminability between two patterns, presumably because of decreased interference between pattern elements (isolation). Experiment 1 also included a second set of tasks, in which the reverse might be expected to occur--decreasing temporal separation (integration) might lead to better discriminability.

Some specific information about the two tasks might help clarify how they favored either isolation or integration. In both tasks, the subjects attempted to discriminate between pairs of patterns. Each pattern consisted of two pattern elements. For both tasks, the same set of pattern elements was used. Representations of these elements are shown in Figure 1. If Pattern Elements 1 and 2 were presented simultaneously, the result would be a rectangular pattern. Similarly, Pattern Elements 3 and 4 and Elements 5 and 6 form the same rectangular pattern. In the first task, the subjects were presented with a pair of patterns that were separated by $750 \mathrm{msec}$. Each member of the pair of patterns consisted of two pattern elements. The elements were selected such that they always formed a rectangle if presented simultaneously. In the first task, the pattern elements were never presented simultaneously but always with brief temporal separations between the two presentations. The subject was required to discriminate between the two patterns. A different trial consisted of two different pairs of pattern elements. A same trial consisted of pairs of identical pattern elements. A representation of same and different trials is shown in Figure 2, panel I. This task was designed to favor isolation of the pattern elements in that, if an integrated pattern is formed (a rectangle) and the pattern elements are unavailable perceptually, subjects will be unable to discriminate between the two patterns.

In the second task, the same pattern elements were used as those in the first task, and subjects were again asked to respond same or different to pairs of patterns made up of these elements. The major difference between the two tasks was how same and different were defined. On a different trial, pattern elements were selected such that at least one member of the pair of patterns did not form a rectangle. A different trial might present Elements 1 and 2 , followed by 1 and 3 . On a same trial, the pattern elements formed a rectangle but, unlike the pattern elements in the first task, the pattern elements differed from one another. Therefore, the pattern elements were not identi- cal on same trials but were identical on the dimension of overall shape. For example, one pattern might be formed from Elements 1 and 2 and the second pattern from Elements 3 and 4 . Because similar pattern elements and temporal separations were used, the initial representations of the patterns should be similar in the two tasks. Whether integration aids or hinders discriminability may depend on how same and different are defined (task demands) and on subsequent processing of the initial representations. In the second task, there were several ways that the subjects could discriminate between these pairs of patterns. It could be on the basis of the difference in the pattern elements. Isolation would favor discriminations on this basis. The subjects could also discriminate on the basis of overall shape-rectangle versus nonrectangle. Several models of the processing of spatial patterns maintain that tactile patterns are subjected to a lowpass spatial filter. The resulting representation is a blurred image of the original spatial pattern (Loomis, 1990). The finer spatial information will tend to be lost, whereas the overall shape of the patterns will be preserved. Integration would favor discriminations on this basis. Also, it has been shown that spatial patterns composed of fewer features-simpler patterns-are more easily processed than are complex patterns (Craig, 1979; Horner, 1991). To the extent that a single (integrated) pattern, such as the rectangle, is less complex than the two pattern elements composing the rectangle, performance would be expected to favor integration over isolation.

\section{Method}

Subjects. All of the subjects were students at Indiana University. They received an hourly wage for their participation. All were experienced in identifying tactile patterns. Nine of the subjects, 6 women and 3 men, participated in the first two sets of measurements. Seven of these subjects, 5 women and 2 men, participated in the third set of measurements.

Apparatus. The apparatus consisted of four components: a tactile display on which the patterns were generated, a visual monitor with which to communicate with the subject, a keyboard on which the subject could respond, and a computer that was used to control the stimuli, to record responses, and to provide information to the subject. An Optacon-type display was used for generating the tactile patterns. The display consisted of 144 tactors arranged in a 6column $\times 24$-row array. Each tactor in the array could be made to vibrate at $230 \mathrm{pps}$. The array measured $1.2 \times 2.7 \mathrm{~cm}$ and fit against the distal portion of the subject's fingerpad. The patterns

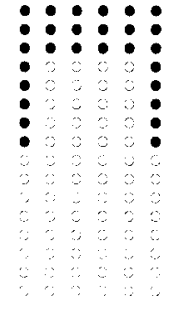

1

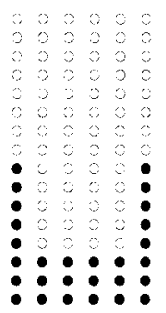

2

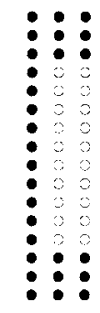

3

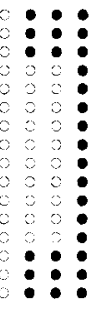

4
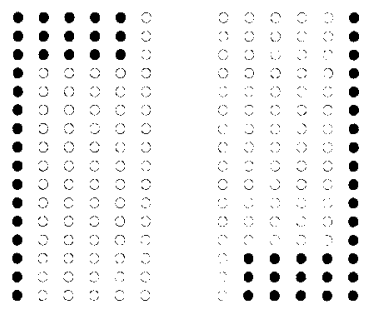

5
6

Figure 1. Representation of the pattern elements. Two pattern elements were presented to create a pattern. 


\section{PATTERN A}

\section{PATTERN B}

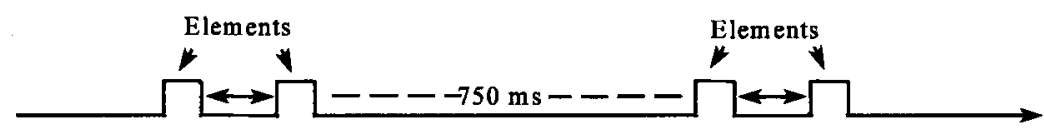

S A M E

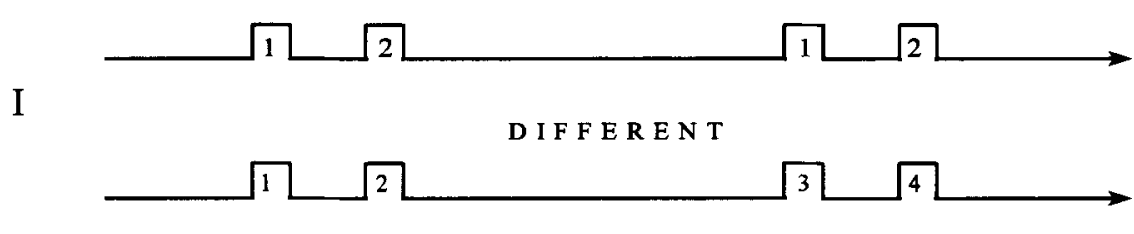

S A M E

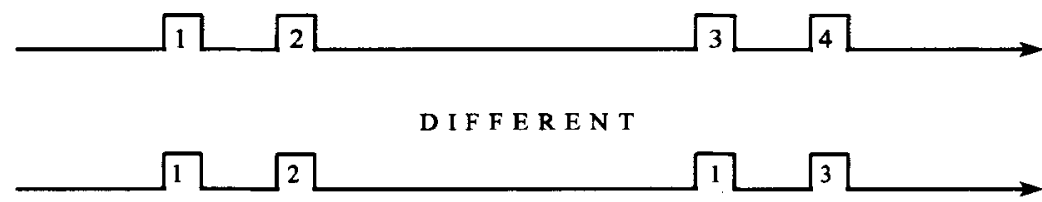

Figure 2. A representation of the experimental paradigm in Experiment 1 (discrimination). Each of the two patterns, A and B, was made up of two pattern elements. The elements were presented at various temporal separations. The time lines marked $I$ are diagrams of the same and different trials in the first set of measurements. The time lines marked $I$ are diagrams of the same and different trials in the second set of measurements. The numbers $1,2,3$, and 4 refer to the pattern elements, as presented in Figure 1.

were confined to the top 18 rows of the display $(1.2 \times 2.0 \mathrm{~cm})$. More details of the apparatus may be found in earlier publications (Craig, 1980; Evans \& Craig, 1992).

Stimuli. Representations of the pattern elements are shown in Figure 1 . The pattern elements were presented for $26 \mathrm{msec}$.

Procedure. There were three sets of measurements. In all of the measurements, the subject's task was to discriminate between two patterns presented sequentially to the left index fingerpad. The patterns were separated by $750 \mathrm{msec}$, as measured from the offset of the trailing pattern element of the first pattern to the onset of the leading pattern element of the second pattern (Figure 2). Each pattern consisted of two pattern elements presented for $26 \mathrm{msec}$. These elements were presented in close succession. Thus, on each trial, the subject received two pattern elements that composed the first pattern and two pattern elements that composed the second pattern. Approximately $50 \%$ of the trials were same and $50 \%$ were different.

In the first set of measurements-the measurements favoring isolation-there were three possible patterns. One pattern was formed by Elements 1 and 2, the second by Elements 3 and 4, and the third by Elements 5 and 6 . The pattern elements were presented successively with SOAs of $26,52,100$, and $300 \mathrm{msec}$. If the elements were presented simultaneously, the three patterns formed would be identical rectangles. On a same trial, the subject received two patterns that consisted of identical pattern elements presented in the same order-for example, 1,2 followed by 1,2 . On a different trial, the subject received two patterns that consisted of a different pair of pattern elements-for example, 1, 2 followed by 3, 4 (Figure 2, panel I).

For the second set of measurements-the measurements favoring temporal integration-the patterns were generated out of the same set of pattern elements, but same trials and different trials were defined differently than they were in the first set of measurements. On a same trial, the pattern elements were selected such that they formed a rectangle, but the two patterns always consisted of different pattern elements-for example, Pattern Elements 1, 2 followed by 3,4 . On a different trial, one of the two patterns was created out of pattern elements that formed a rectangle, whereas the other pattern was created out of pattern elements that did not form a rectangle. The other pattern was composed of Pattern Elements 1,$3 ; 2,4$; or 4,6, (Figure 1). A representation of same and different trials is shown in Figure 2, Panel II. SOAs of 0, 26, 52, 100 , and $300 \mathrm{msec}$ were tested. The pattern elements were $26 \mathrm{msec}$ in duration.

In a third set of measurements, the procedure that had been followed for the second set of measurements was modified in order to improve discriminability and to increase the range of performance levels. The duration of presentation was increased from 26 to $52 \mathrm{msec}$. The height of the patterns was reduced to eight rows in order to make the four sides more equal in length. The task was identical to that in the second set of measurements. SOAs of 0,52 , 100 , and 300 msec were tested.

For all sets of measurements, the subjects were tested individually. Each was seated with his or her left arm resting on the table in front of him or her. The subject's left index fingerpad contacted the tactile array. The subject responded with his or her right hand, using a keyboard with keys marked $\mathrm{S}$ for same and $\mathrm{D}$ for different. The intensity of vibration was set at a comfortable level, $36 \mathrm{~V}$ to the driving circuits, which produced a moderately intense pattern. The trials were self-paced. The subject initiated a trial by pressing a key on the keyboard. The first pattern was presented $1 \mathrm{sec}$ later and consisted of two pattern elements. After the second pattern, 
the subject responded and received feedback in the form of same or different. Trials were blocked by SOA. The blocks of 50 trials were tested in random order. The subjects wore earplugs and earphones, through which low-pass filtered noise was presented in order to eliminate auditory cues from the tactile display. For the first set of measurements, the subjects were tested for four blocks of trials; for the second set of measurements, for five blocks of trials; and for the third set of measurements, for four blocks of trials.

\section{Results and Discussion}

The results from the first two sets of measurements are shown in Figure 3. As in previous studies of discrimination (Cholewiak \& Craig, 1984; Craig, 1983; Horner \& Craig, 1989), the responses on same and different trials were converted to $d^{\prime}$ and then maximum percentage correct, $P(\mathrm{C})$ max (McFadden, 1970). It is $P(\mathrm{C})$ max that is plotted as a function of SOA. For the first set of results marked isolation, 2 of the 9 subjects were essentially at chance, and the results from these 2 subjects were not used in the data analysis. For the results marked integration, the same 2 subjects were at chance or just above chance, and the results from these subjects were not used in the data analysis. The results from the first task, the one favoring isolation, show a clear improvement in discriminability as the temporal separation of the pattern elements increases. A dashed line has been added that connects the point at the 26-msec SOA to a point at $0 \mathrm{msec}$ set at $50 \%$ performance. For pairs of patterns at $0-\mathrm{msec} \mathrm{SOA}$, the same and different patterns are identical. Both patterns would be identical rectangles, and, therefore, performance would be at chance. A one-way analysis of variance (ANOVA) showed a significant effect of SOA $[F(3,18)=30.09, p<.0001]$. The results shown in Fig- ure 3 are typical of those reported in a number of studies of temporal masking with spatial patterns: Performance improves rapidly as the temporal separation is increased to $100 \mathrm{msec}$ and then more gradually with greater temporal separations (Craig \& Evans, 1987; Evans \& Craig, 1986; Kirman, 1984). The information required for discriminating between the two patterns is contained in both pattern elements. It is likely that the function shown in Figure 3 (isolation) is the result of mutual interference between the two pattern elements and is attributable to both forward and backward masking.

The results from the second task, the one favoring integration, show performance declining as SOA increases from 0 to $100 \mathrm{msec}$ (Figure 3, integration). Beyond $100 \mathrm{msec}$, performance appears to rise again. Out to $100 \mathrm{msec}$, the two functions shown in Figure 3 appear to mirror one another. This is to be expected if temporal integration is affecting the pattern elements - in one case, hindering discrimination and, in the other case, aiding it. The ANOVA shows a significant effect of SOA $[F(4,24)=$ $9.70, p<.0001]$.

As noted before, Mahar and Mackenzie (1993) found only decrements in performance as the time between pattern elements decreased, even under conditions that might be expected to favor temporal integration. One reason for their findings might be the time intervals that they tested. The briefest interval that they used was $94 \mathrm{msec}$. For the purpose of comparison with the present results, the results from their integration condition were converted to $P(\mathrm{C})$ max from the $d^{\prime}$ values that they reported. $P(\mathrm{C})$ max at the $94-\mathrm{msec} \mathrm{SOA}$ was $70 \%$ correct; at $205 \mathrm{msec}, 76 \%$; and at $450 \mathrm{msec}, 76 \%$. In the integra-

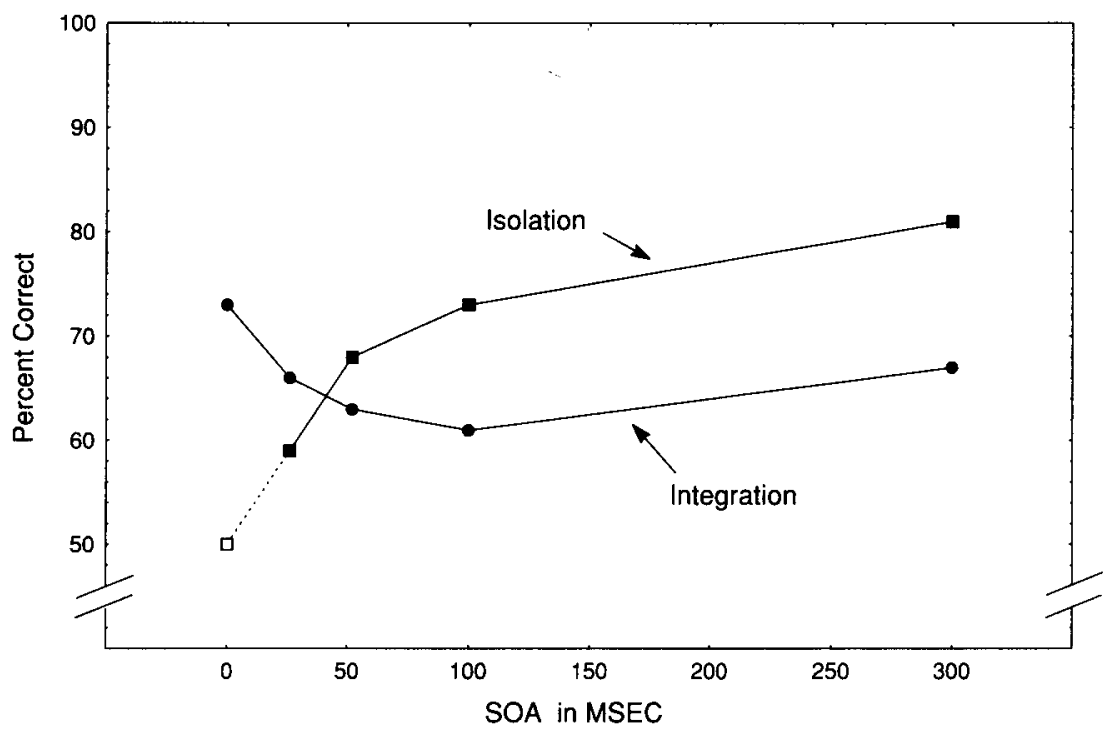

Figure 3. The results from the discrimination measurements showing maximum percentage correct as a function of the stimulus onset asynchrony (SOA) between pattern elements. The function labeled isolation represents the results from the first set of measurements. A point at $50 \%$ correct has been added at the 0-msec SOA to indicate what chance performance would have been, had the two pattern elements been presented simultaneously. The function marked integration represents the results from the second set of measurements. 
tion function shown in Figure 3, the percent correct at the 100 -msec SOA was $61 \%$, increasing to $66 \%$ at the $300-\mathrm{msec}$ SOA. Over a comparable range of SOAs, performance improved in a similar manner for both the present data and Mahar and Mackenzie's results. To the extent that temporal integration is a low-level process that affects the initial representation of vibrotactile patterns, one would expect to see similar results even with quite different stimulus configurations. Mahar and Mackenzie observed that, had they been able to examine briefer SOAs, they might have seen an increase in performance levels and found support for the improvement of pattern perception by temporal integration. The present results support this observation.

The range of performance in the second set of measurements was fairly limited. The percent correct at $0 \mathrm{msec}$ was $74 \%$, decreasing to $61 \%$ correct at $100 \mathrm{msec}$. As noted, in an attempt to improve discriminability and to increase the range of performance levels, a third set of measurements was made with a modified set of patterns. The results from these measurements-also favoring integration-are shown in Figure 4. One of the subjects was at chance, and her data were not included. The range of performance was increased slightly, from $82 \%$ correct at $0 \mathrm{msec}$, dropping to $64 \%$ correct at $52 \mathrm{msec}$. The overall function is similar to that seen in Figure 3, integration. The major difference is that the duration over which integration appears to operate has decreased from 100 - to 52-msec SOA.

For the integration data, the increase in performance at the longest SOA is of particular interest. If the difference is reliable, it suggests that the subjects changed the way in which they performed this discrimination task as a function of the temporal separation between pattern elements. Beyond 52-to-100-msec SOAs - the approximate temporal interval over which integration is seen - subjects may attempt to perform the task by attending to the individual pattern elements. As in the isolation task, the greater temporal separation would lead to better discriminability. The Tukey HSD test on both sets of data provided partial support for this view. For the first set of integration data (Figure 3), there was no significant difference between the results at $100 \mathrm{msec}$ and those at $300 \mathrm{msec}(p=.07)$; however, for the second set of integration data (Figure 4), there was a significant difference between the results at $100 \mathrm{msec}$ and those at $300 \mathrm{msec}$ $(p<.05)$.

The main findings from Experiment 1 are (1) that there are conditions under which the discrimination of tactile spatial patterns appears to be aided by temporal integration, (2) whether or not integration or isolation benefits discrimination depends on the patterns and pattern elements and the temporal separation between pattern elements, and (3) that the integration and isolation functions appear to mirror one another at brief SOAs.

\section{EXPERIMENT 2}

The aim of Experiment 2 was to see the extent to which the discrimination results from Experiment 1 could be used to predict results in an identification task. In one set of measurements in Experiment 2, the subjects identified spatial patterns composed of the same set of pattern elements as those in Experiment 1. The pattern elements, the way in which the elements were combined, and the resulting patterns to be identified are shown in Figure 5. Presenting subjects with two pattern elements, such as those used in Experiment 1, should produce the same initial representation, whether the task is discrimination or identification. Integration should be maximal

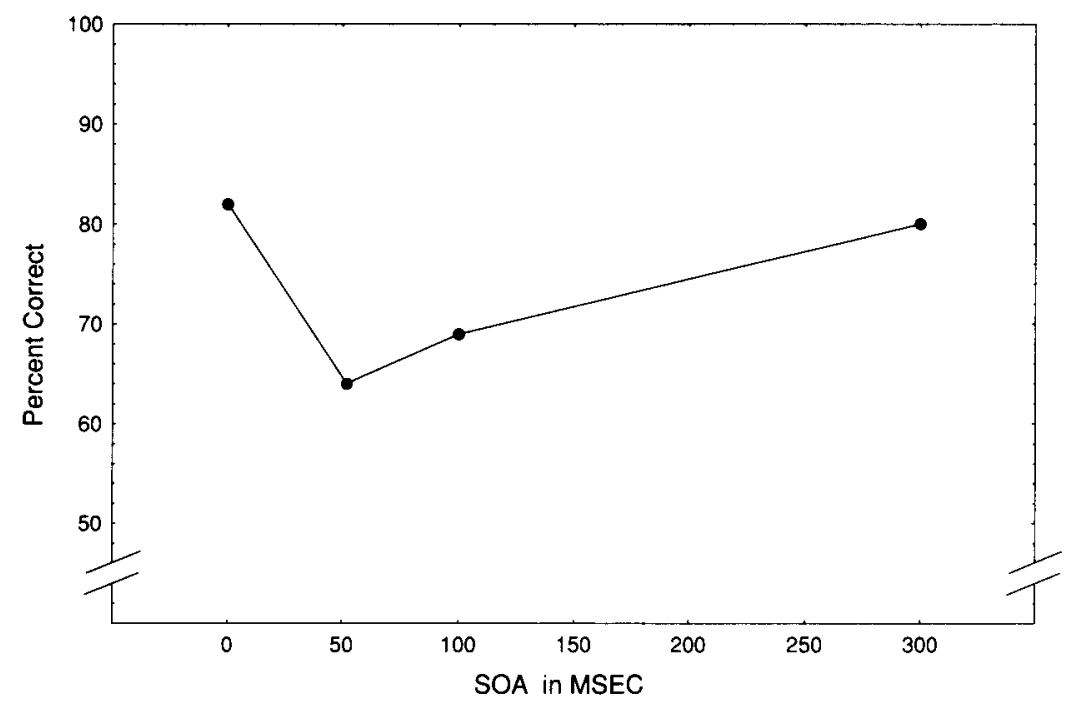

Figure 4. The results from the third set of discrimination measurements showing maximum percentage correct as a function of the stimulus onset asynchrony between pattern elements. The patterns were similar to those used to produce the integration results shown in Figure 3. 


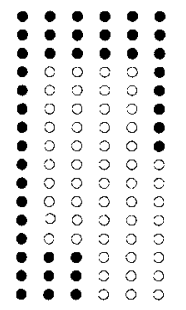

1
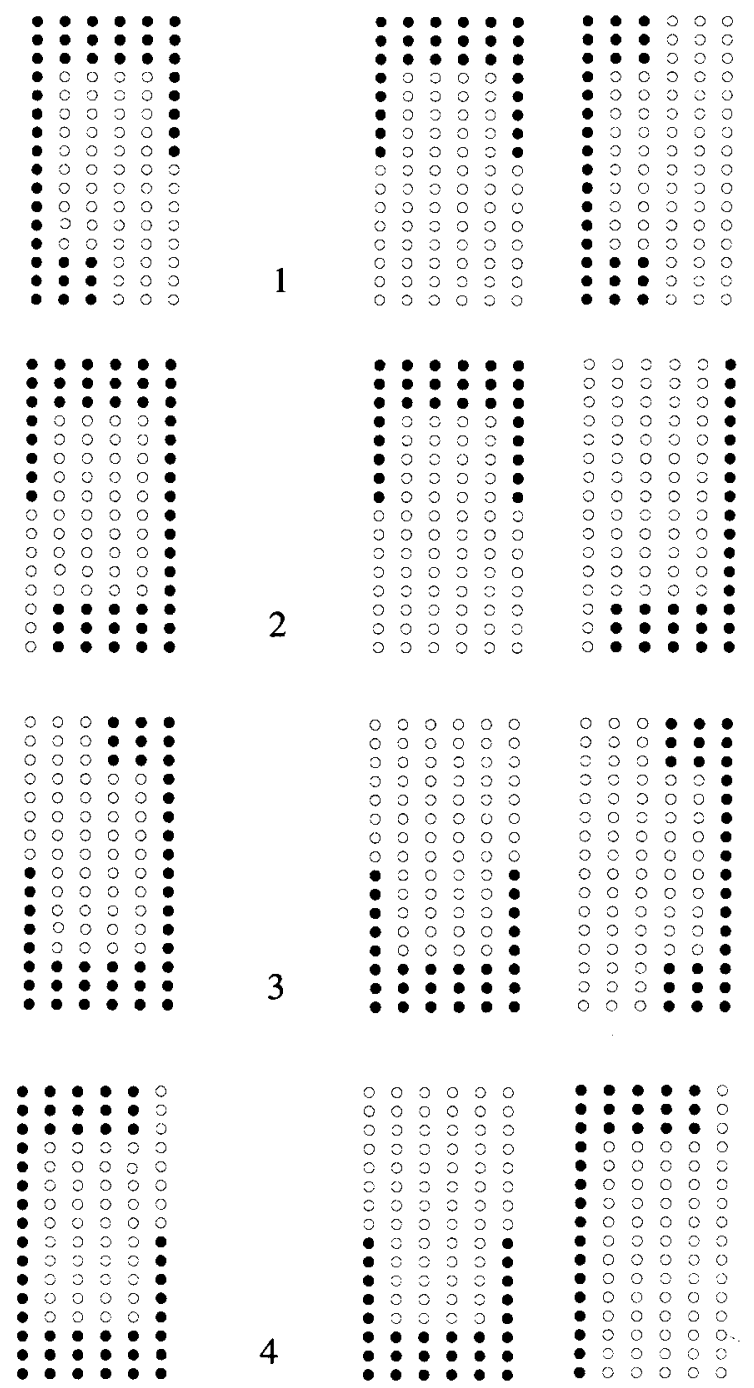

\section{Msec SOA}

\section{Pattern Elements}

Figure 5. Representations of the patterns and pattern elements in Experiment 2 (identification).

at brief SOAs and decline to $100-\mathrm{msec}$ SOA, and isolation should reflect the opposite function. However, it is likely that further processing of the initial representation and the effect that this has on identification performance would be affected by a number of additional variables.

In an identification task, subjects must relate the initial representation of the pattern to a representation stored in long-term memory. All other things being equal, one would expect that more familiar patterns would be easier to identify than less familiar patterns. In fact, that is one reason that Mahar and Mackenzie (1993) suggested using a discrimination task -it avoids some of the problems associated with the differential experiences that subjects may have had with patterns. The nature of the experiences that subjects have had with tactile spatial patterns might favor integration. In the study mentioned earlier with letters of the alphabet (Craig, 1982), identification performance was good when the letter halves were presented simultaneously or with a very brief temporal separation between them. Performance declined at longer separations. The subjects had had some tactile experience identifying the letters and extensive visual experience with letters. All of the experience was, however, with the pattern elements presented simultaneously and virtually none with letter halves presented successively. This experience, both visual and tactile, should aid the development of the patterns' representations in memory. Although limited, the tactile experience by itself would help subjects link the initial representation of the tactile input with the corresponding representation in memory. The use of patterns that are relatively unfamiliar (Figure 5) should provide a more unbiased estimate of the role of integration/isolation in pattern identification.

Given that subjects have had little or no experience with identifying the patierns and pattern elements shown in Figure 5, would performance follow a temporal integration or temporal isolation function? One would expect that the overall shape of the patterns would be an important determinant of performance. On that basis, at brief SOAs, subjects would err in selecting among similarly shaped rectangular patterns. At longer SOAs, subjects will have less difficulty identifying the isolated pattern elements whose overall shapes differ from one another. Some pattern identification results support the idea that overall shape is important and, further, that subjects have difficulty detecting gaps in continuous figures. Using an Optacon-type display, it has been shown that subjects frequently respond $O$ when presented with the letter $C$ (Craig, 1979). The critical feature for identifying the rectangular patterns is the location of the gap. This difficulty might lead to poor performance at $0 \mathrm{msec}$ with the rectangular patterns, with performance improving at longer SOAs, when the identification can be based on the pattern elements.

Because one set of measurements in Experiment 2 followed an isolation function, we repeated the earlier set of letter measurements that had followed the opposite function, an integration function. In this replication, we wanted to follow a procedure that more nearly matched that with the rectangular patterns. In the earlier study with the letters BEMRW, the patterns were divided in half in four different ways--along the horizontal axis, the vertical axis, and the two diagonals. Dividing the patterns in these ways and randomly selecting which half was presented first produced eight different patterns for each letter, except when the two halves were presented simultaneously-that is, at the $0-\mathrm{msec}$ SOA. At the 0 -msec SOA, the patterns could be generated in only one way. The results, as noted before, were that identification performance was best at the 0 -msec SOA and declined gradually with increasing SOA (Craig, 1982). This result supported the idea that temporal integration can benefit pattern 
perception. It could also be argued, however, that performance at the 0-msec SOA was superior to performance at the other conditions - that is, the longer SOAs - because, at these longer SOAs, there were more possible patterns (eight) to be learned. To provide a better basis for comparison with rectangular patterns, the measurements from the earlier study were repeated, while the number of possible patterns at each SOA, including $0 \mathrm{msec}$, were kept constant.

A third set of measurements were taken to examine how well the subjects could identify the pattern elements that composed the patterns in the first two sets of measurements. At longer SOAs, beyond the limits of temporal integration, subjects have to process the pattern elements individually. The relative distinctiveness of the pattern elements should predict how well subjects identify the patterns at these longer SOAs.

\section{Method}

Subjects. The subjects who were tested in Experiment 2 were similar to those in Experiment 1. For the letter patterns in the first set of measurements, 4 women and 1 man were tested. With the rectangular patterns, 3 women and 3 men were tested. In the third set of measurements, which involved the identification of pattern elements, 7 subjects, 5 women and 2 men, were tested.

Apparatus. The same apparatus as that in Experiment 1 was used in Experiment 2.

Procedure. A major difference in procedure between Experiments 1 and 2 was that the subjects were required to identify the patterns rather than to discriminate between two patterns. The subjects initiated each trial and, $1 \mathrm{sec}$ later, received a pattern. In the first two sets of measurements, the pattern was composed of two pattern elements. The pattern elements were presented successively at SOAs of $0,26,52,100$, and 300 msec. The trials were blocked by SOA. Each block consisted of 50 trials. The five SOAs were tested in random order during each session. Each set of patterns was tested for six sessions. The subjects responded by press- ing keys marked with visual representations of the patterns. The subjects received trial-by-trial feedback in the form of the word correct or of the number of the correct pattern, if they were incorrect.

Two sets of patterns were tested. In the first set, five letters, BEMRW, were tested. These were the same five letters that had been tested in an earlier study of temporal integration (Craig, 1982). They were selected because they were among the most difficult letters to identify (Craig, 1979). The pattern elements for each letter were created by dividing the letter in half along the positive diagonal.

In the second set of measurements, the patterns (Figure 5) were generated from the pattern elements in Experiment 1. As in the first set of measurements, the subject's task was to identify which one of the patterns had been presented. For both sets of measurements, the two elements making up the patterns were always presented in the same order.

In the third set of measurements, the subjects identified the pattern elements. One stimulus set was formed by using the 10 pattern elements that made up the letters BEMRW. The other stimulus set was formed by using the 6 pattern elements in Experiments 1 and 2 (Figure 1). The subject's task was to identify which pattern element had been presented. The subjects responded by means of a keypad labeled with visual representations of the pattern element. On alternate blocks of trials, the subjects were presented with either the letter elements or rectangular pattern elements. The elements were present singly for a duration of $26 \mathrm{msec}$. Trial-by-trial feedback was provided. The subjects were tested for six 50-trial blocks for each stimulus set.

\section{Results and Discussion}

The data from the letter patterns are shown in Figure 6 . These results replicate the results from the earlier study (Craig, 1982). Performance drops off fairly rapidly from the 0 - to the 100 -msec SOA and then more gradually out to $300 \mathrm{msec}$. A one-way ANOVA showed a significant effect of SOA $[F(4,16)=22.93, p<.0001]$. These results are what one would predict if, as SOA decreases, subjects were increasingly able to combine the pattern

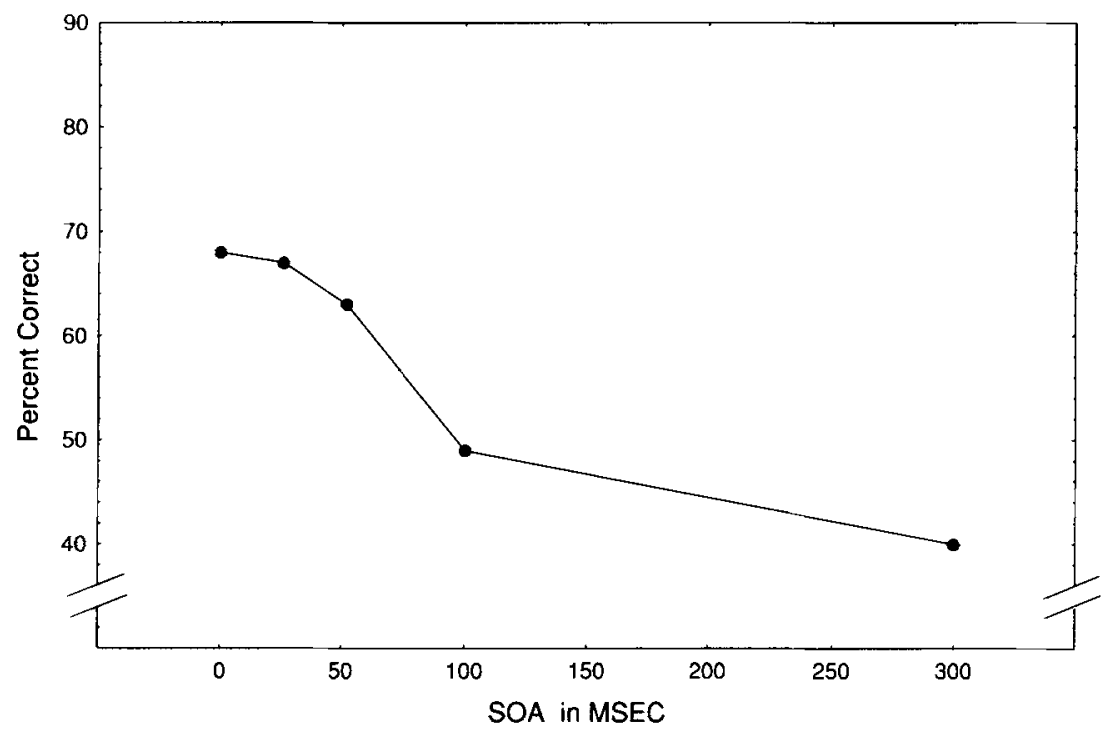

Figure 6. The results from the letter patterns (BEMRW), showing the percentage of correct identifications as a function of the stimulus onset asynchrony between pattern elements. 
elements into a single percept by means of temporal integration. At the same time, interference between the two pattern elements will also be increasing as SOA decreases. That is, if the subjects were asked to identify one of the pattern elements rather than the patterns themselves, performance in this task should be poor at brief SOAs.

In an earlier study with these five letters, the pattern elements could be presented in any one of eight different combinations for each letter. The similarity of the present results, in which there was only one combination of pattern elements for each letter, to the earlier results indicates that the number of combinations of pattern elements does not affect the temporal integration function. Equating the number of combinations of pattern elements at the 0-msec SOA (one) with the number at the longer SOAs (one) has no noticeable effect on the temporal integration function. The higher performance level at $0 \mathrm{msec}$ does not appear to be due to the subjects having to learn fewer combinations when the pattern elements are presented simultaneously.

The results from the second set of measurements, shown in Figure 7, present quite a different picture than do those from the first measurements. Here, as expected, performance improves with increasing SOA. The ANOVA showed a significant effect of SOA $[F(4,20)=9.46, p<$ $.001]$. The tasks for both sets of measurements appear to be the same - to identify a spatial pattern composed of two pattern elements-yet the functions differ from one another.

The function relating percent correct to the time between pattern elements appears to be similar to the isolation function in Figure 3 that was generated in a discrimination task. One of the goals of Experiment 2 was to see the extent to which identification performance could be predicted by discrimination performance when the same pattern elements were used. To compare the two functions, the results were reanalyzed, and the change in percent correct from the 0 -msec condition was calculated. Specifically, the differences between the percent correct at the $0-\mathrm{msec}$ SOA in the discrimination (isolation) task (assumed to be 50\% correct, or chance) and at the remaining four SOAs were calculated. Similarly, the differences between the percent correct at the $0-\mathrm{msec}$ SOA $(53 \%$ correct) in the identification task (Figure 7$)$ and at the remaining four SOAs were calculated. These changes in percent correct were plotted as a function of SOA and are shown in Figure 8. The logarithmic regression function provides a reasonably good fit to the data $(r=.95)$. The results indicate that, for both discrimination and identification, percent correct is a linear function of the logarithm of the temporal separation between the pattern elements. The similarity between the two functions supports the idea that temporal integration is a low-level process. The initial representation of the pattern elements does not change as a function of the task. It is the same for a discrimination task as it is for an identification task.

The results with the rectangular patterns (Figure 7) are consistent with the view that the overall shape of the patterns is an important determinant of performance. At brief SOAs, the pattern elements are integrated to form four rectangles that are similar in shape to one another. At 300-msec separation, it is unlikely that temporal integration is contributing much to the identification of the pattern. Thus, performance should be based on the distinctiveness of the pattern elements. Identification performance with the rectangular patterns rose to $86 \%$ cor-

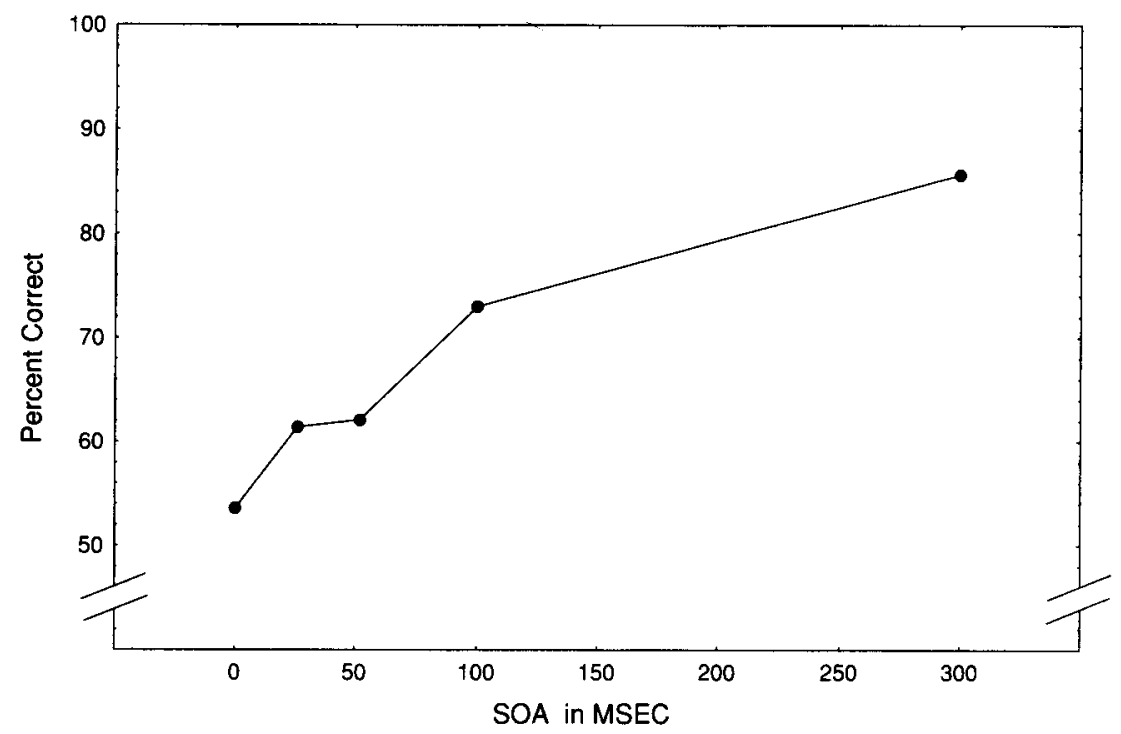

Figure 7. The results from the patterns represented in Figure 5, showing the percentage of correct identifications as a function of the stimulus onset asynchrony between pattern elements. 


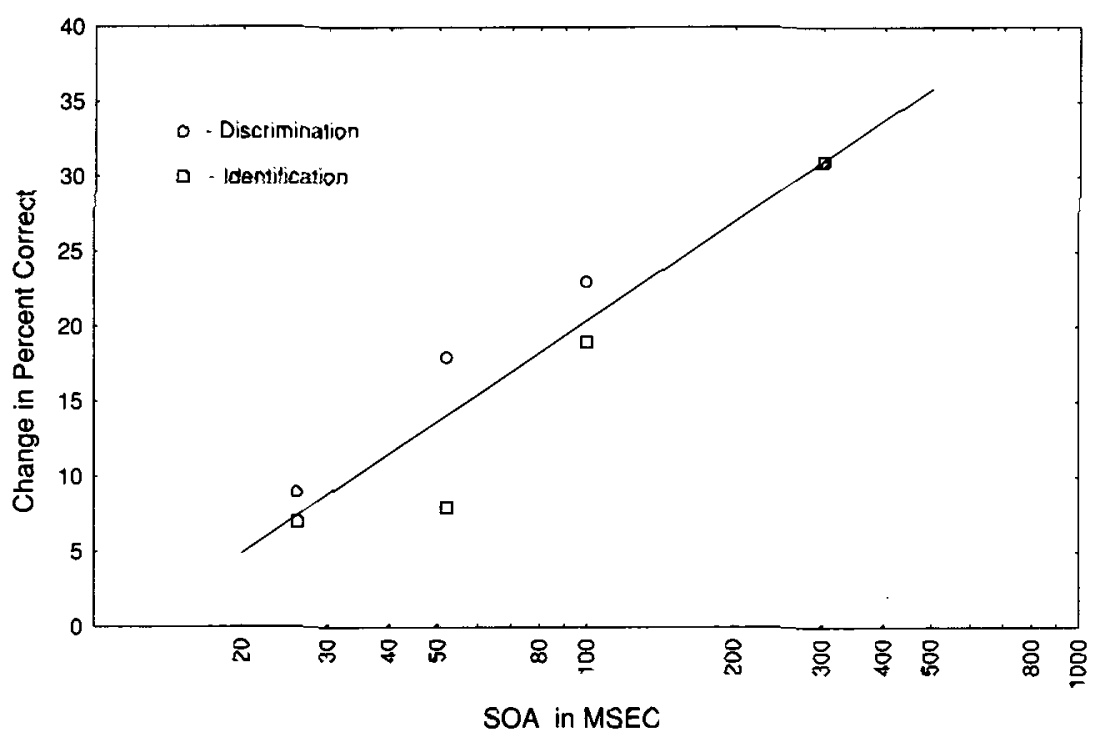

Figure 8. The discrimination results from Figure 3 (isolation) and the identification results from Figure 7 are replotted to show the change in percent correct from performance at 0-msec stimulus onset asynchrony (SOA) as a function of the logarithm of the SOA between pattern elements. A single function has been fit to the data $(r=.95)$.

rect, at the $300-\mathrm{msec} \mathrm{SOA}$ (Figure 7), whereas, with the letter patterns, performance fell to $40 \%$ correct (Figure 6). The results from the third set of measurements, in which the subjects identified the pattern elements, are consistent with the view that the distinctiveness of the pattern elements determines performance at the longer SOAs. The percent correct for the letter halves was $37 \%$, and for the rectangular pattern elements, it was $76 \%$. At longer SOAs, the subjects appear to be basing their identification decision on the individual pattern elements and not on the integration of the elements, at least not at a sensory level. It is possible that the information about the two elements might be combined at a later stage of processing and that that leads to improved performance.

In addition to considering the distinctiveness of the pattern elements, one needs to consider the distinctiveness of the patterns formed from the integration of the pattern elements. Pattern sets can be created such that, at the 0 -rnsec SOA, identification is nearly impossible; yet, when divided into pattern elements, identification performance might be quite good. Suppose that a set of rectangular patterns differed only by the location of a gap created by just a single tactor. At the $0-\mathrm{msec}$ SOA, the patterns would be virtually indistinguishable; however, the pattern elements could be selected so that each pattern was associated with quite distinct pattern elements, such as (1) the top and bottom of the rectangle and (2) the left and right sides. Both patterns would also differ by the location of the one-tactor gap; however, only at longer temporal separatjons between pattern elements would it be likely that subjects would be above chance in identifying such patterns.

\section{GENERAL DISCUSSION}

In theory, temporally separated pattern elements always contain sufficient information to perceive a spatial pattern. Any stimulus manipulation that leads to greater distinctiveness of the pattern elements shouid improve the perceptibility of a pattern, all other things being equal. Studies of masking have shown that increasing the temporal separation between two patterns (and, by extension, pattern elements) reduces mutual interference and increases the perceptibility of the patterns (Craig \& Evans, 1987; Evans, 1987; Evans \& Craig, 1986). This being the case, the major question is, why does performance improve under some circumstances in which the temporal separation between pattern elements is reduced? One answer is that reducing the temporal separation between pattern elements increases the likelihood that the elements wi!! be integrated into some kind of perceptual whole. For this reason, the focus of the present study has been on temporal integration, and the question has been rephrased to ask, why does temporal integration improve pattern perception? One reason is that subjects may have greater familiarity with the patterns presented as a whole than with the pattern elements. Another reason may be that less complex patterns - those composed of fewer line segments -are, in general, easier to identify and discriminate than more complex patterns (Craig, 1979; Horner, 1991). Thus, for the letters, the integrated pattern would be simpler than the two patterns treated separately: The two pattern elements contain more line segments than does the integrated letter. When the letters were divided, two line segments 
were often created from a single line segment. Of course, the same is true for the rectangular patterns, where integration led to poorer performance. Identification performance at the $0-\mathrm{msec}$ SOA for the rectangular patterns was poor ( $53 \%$ correct), as compared with identification performance with the more complex letter patterns at the 0 -msec SOA ( $68 \%$ correct). Thus, although the rectangular patterns might appear to be simpler than the letters, performance on these patterns is, in fact, worse. With the rectangular patterns, integration produces patterns that are similar in overall shape and, thus, difficult to identify. Also, the tendency to perceive a closed figure-the Gestalt principle of closure-will cause difficulty in distinguishing among the possible patterns.

The importance of shape and the principle of closure might also help to explain the discrimination results. For the discrimination results shown in Figures 3 and 4, the briefest SOAs produced rectangular-shaped patterns. If these were visual stimuli, one might assume that the rectangle was a simpler figure and, thus, more readily perceived than the elements composing the pattern. The tendency to perceive a rectangle when the pattern elements were presented at brief temporal separations would improve performance when the task was to discriminate a rectangular from a nonrectangular shape (Figure 3 [integration] and Figure 4). By the same token, when the task was to discriminate one rectangular pattern from another on the basis of the elements making up the rectangle, the tendency to perceive a rectangle would hinder discriminability (Figure 3 [isolation]).

One might expect temporally integrated patterns to be easier to perceive than temporally isolated patterns, because the number of patterns to be learned is reduced. As the fingerpad is moved across a spatially extended pattern, the number of pattern elements could conceivably be quite large. A raised letter, a Braille pattern, or the edge of a key could be broken down into a number of different pattern elements. Temporal integration would tend to yield a single percept, easier to encode, easier to remember. Having said that, the results from the letter identification task (Figure 6) offer little support for the idea that reducing the number of elements improves performance at nonzero temporal separations. As noted before, in an earlier study, each letter was divided into eight possible pairs of pattern elements (Craig, 1982). The earlier results showed no lower levels of performance; indeed, they showed somewhat higher levels of performance, at nonzero SOAs, than those presented in Figure 6, where each letter was divided into only one pair of pattern elements. One way to view these results is that, at the briefer SOAs, subjects are in effect dealing with a single, integrated pattern. The number of elements is irrelevant, because the same integrated pattern is produced by all pattern elements.

In considering how the present results might apply to haptic exploration in general, several points need to be kept in mind. First, the pattern elements are highly re- producible in the laboratory setting but are more highly variable in haptic exploration. As the finger is moved over an object, the patterns will change, depending on the orientation of the fingerpad relative to the object. Slight changes in orientation can produce large changes in how the pattern elements are generated sequentially across the fingerpad. Also, the rate at which the hand is moved will change the temporal separation between pattern elements. What is likely to be more stable in such explorations is the spatial pattern itself. Thus, the highly reproducible patterns generated in the laboratory may lead to better performance with the temporally separated pattern elements and, in turn, lead to an underestimation of the effectiveness of temporal integration.

The general issue of integration/isolation developed out of a concern with temporal masking. The task in studies of temporal masking typically forces a subject to attempt to isolate the target from the nontarget, and, thus, it is not surprising that temporal masking functions resemble isolation functions (Kirman, 1973; Mahar \& Mackenzie, 1993). Several studies have also suggested that, in addition to integration's interfering in target identification, response competition may also cause interference (Craig, 1995; Craig \& Evans, 1995). According to a response competition view of interference, both the target and the nontarget are processed to the point of evoking responses. The subject errs by responding with the wrong pattern. Response competition as a mechanism implies that subjects can isolate the target and nontarget sufficiently, so that subjects can respond with either one. It has been suggested (Craig, 1996; Mahar \& Mackenzie, 1993 ) that the integrated percept retains a relatively faithful representation of both the target and the nontarget: As noted before, subjects often respond with a composite pattern, not simply randomly. Such an integrated pattern may contain sufficient information for identifying either the target or the nontarget. The time course of response competition-maximum at brief temporal separations and much reduced beyond $100-\mathrm{msec}$ SOAs - parallels the integration/isolation function in the present study. In short, the present results are consistent with current views of temporal masking.

\section{REFERENCES}

Cholewiak, R. W., \& Craig, J. C. (1984). Vibrotactile pattern recognition and discrimination at several body sites. Perception $\&$ Psvchophysics, 35, 503-514.

CRAIG, J. C. (1979). A confusion matrix for tactually presented letters. Perception \& Psychophysics, 26, 409-411.

CRAIG, J. C. (1980). Modes of vibrotactile pattern perception. Journal of Experimental Psychology: Human Perception \& Performance, 6 , 151-166.

Craig, J. C. (1982). Temporal integration of vibrotactile patterns. Perception \& Psychophysics, 32, 219-229.

Craig, J. C. (1983). The role of onset in the perception of sequentially presented vibrotactile patterns. Perception \& Psychophysics, $\mathbf{3 4}$, 421-432.

Craig, J. C. (1995). Vibrotactile masking: The role of response competition. Perception \& Psychophysics, 57, 1 190-1200. 
CRAIG, J. C. (1996). Interference in identifying tactile patterns: Response competition and temporal integration. Somatosensory \& Motor Research, 13, 199-213.

Craig, J. C., \& Evans, P. M. (1987). Vibrotactile masking and the persistence of tactual features. Perception \& Psychophysics, 42, 309317.

Craig, J. C., \& Evans, P. M. (1995). Tactile selective attention and temporal masking. Perception \& Psychophysics, 57, 511-518.

Evans, P. M. (1987). Vibrotactile masking: Temporal integration, persistence, and strengths of representations. Perception \& Psychophysics, 42, 515-525.

Evans, P. M., \& Craig, J. C. (1986). Temporal integration and vibrotactile backward masking. Journal of Experimental Psychology: Human Perception \& Performance, 12, 160-168.

Evans, P. M., \& Craig, J. C. (1992). Response competition: A major source of interference in a tactile identification task. Perception \& Psychophysics, 51, 199-206.

HORNER, D. T. (1991). The effect of complexity on the perception of vibrotactile patterns. Perception \& Psychophysics, 49, 551-562.

Horner, D. T., \& Craig, J. C. (1989). A comparison of discrimination and identification of vibrotactile patterns. Perception \& Psychophysics, 45, 21-30.

KIRMan, J. H. (1973). Tactile communication of speech: A review and analysis. Psychological Bulletin, 80, 54-74.

KIRMAN, J. H. (1984). Forward and backward tactile recognition masking. Journal of General Psychology, 111, 83-99.

LoOMIs, J. M. (1990). A model of character recognition and legibility. Journal of Experimental Psychology: Human Perception \& Performance, 16, 106-120.

MAHAR, D. P., \& MACKENZIE, B. D. (1993). Masking, information integration, and tactile pattern perception: A comparison of the isolation and integration hypotheses. Perception, 22, 483-496.

MCFADDEN, D. (1970). Three computational versions of proportion correct for use in forced-choice experiments. Perception \& Psychophysics, 8, 336-342.

(Manuscript received August 2, 1996;

revision accepted for publication June 29,1997 .) 